\title{
Yasmina Wicks, Javier Álvarez-Mon. “Grave matters in the Zagros mountains: the Lama cemetery, grave 69 and its cylinder seal re- evaluated in an Elamite context"
}

\section{Rémy Boucharlat}

\section{OpenEdition}

\section{Journals}

Édition électronique

URL : https://journals.openedition.org/abstractairanica/53145

DOI : 10.4000/abstractairanica. 53145

ISSN : 1961-960X

Éditeur :

CNRS (UMR 7528 Mondes iraniens et indiens), Éditions de l'IFRI

Référence électronique

Rémy Boucharlat, « Yasmina Wicks, Javier Álvarez-Mon. "Grave matters in the Zagros mountains: the Lama cemetery, grave 69 and its cylinder seal re-evaluated in an Elamite context" », Abstracta Iranica [En ligne], Volume 42-43 | 2021, document 7, mis en ligne le 30 décembre 2021, consulté le 13 décembre 2022. URL : http://journals.openedition.org/abstractairanica/53145; DOI : https://doi.org/ 10.4000/abstractairanica.53145

Ce document a été généré automatiquement le 13 décembre 2022.

Tous droits réservés 


\title{
Yasmina Wicks, Javier Álvarez-Mon. "Grave matters in the Zagros mountains: the Lama cemetery, grave 69 and its cylinder seal re- evaluated in an Elamite context"
}

\author{
Rémy Boucharlat
}

\section{RÉFÉRENCE}

Yasmina Wicks, Javier Álvarez-Mon. "Grave matters in the Zagros mountains: the Lama cemetery, grave 69 and its cylinder seal re-evaluated in an Elamite context" (with a contribution on seal no. 69.07.251 by Gian Pietro Basello), Oriens Antiquus Series Nova 2, 2020, p. 99-115.

1 Parmi plus de cinquante tombes fouillées dans le cimetière de Lama dans le HautKarun, province du Kohgiluyeh-o Boyer-Ahmad, à $50 \mathrm{~km}$ au nord de Yasuj, une seule tombe ( $\left.{ }^{\circ}{ }^{\circ} 69\right)$ contenait un sceau-cylindre. Il est important de noter que la tombe a été maintes fois réutilisée sur une longue période comme en témoignent les multiples inhumations, le mobilier funéraire à l'intérieur et les restes d'offrandes à l'extérieur : tombe d'une famille ou d'un groupe social ? Le sceau représente un homme armé d'un arc poursuivant une autruche et sur une face il est porte quelques signes cunéiformes disposés sur cinq registres. Par les signes inscrits, les fouilleurs avaient daté ce sceau du milieu ou de la deuxième moitié du II ${ }^{e}$ mill. av. n.è, tout en notant que l'image, proche par le style du Néo-Elamite II et de l'art néo-assyrien, avait pu être ajouté au vIII siècle.

2 L'un des auteurs du présent article J.A-M. avait déjà contesté cette date dans son abstract sur un rapport des fouilleurs (AI 37-38-39, 3.2.1 $\mathrm{n}^{\circ}$ 9), proposant provisoirement le $\mathrm{VII}^{\mathrm{e}}$ ou le $\mathrm{VI}^{\mathrm{e}}$ siècle aussi bien pour l'inscription que pour la scène 
représentée. C'est ce que confirme l'analyse précise conduite dans cet article. On remarque que parmi d'autres éléments de comparaisons sont évoqués les tablettes de l'Acropole et le sceau de Kurash.

3 Suivent des observations intéressantes d'abord sur le mode de vie des populations du cimetière. Les A sont prudents avant de parler de pasteurs nomades, comme beaucoup de chercheurs l'écrivent trop rapidement pour le Zagros et du fait de l'absence apparente de villages de sédentaires à proximité ; a contrario l'abondance de la poterie dans les tombes n'est pas une caractéristique des nomades. Élargissant le propos, les A s'interrogent sur le contrôle qu'exerçait la puissance élamite dans cette région. Cette mainmise est attestée au II millénaire, plus incertaine, mais probable au début du I ${ }^{\mathrm{er}}$ mill., en dépit du morcellement politique élamite et la fluctuation des frontières orientales dans le Zagros et vers le Fars.

\section{AUTEURS}

\section{RÉMY BOUCHARLAT}

UMR 5133 CNRS-Université de Lyon 